\title{
The illusion of knowing: Failure in the self-assessment of comprehension
}

\author{
ARTHUR M. GLENBERG, ALEX CHERRY WILKINSON, and WILLIAM EPSTEIN \\ University of Wisconsin, Madison, Wisconsin 53706
}

\begin{abstract}
The illusion of knowing is the belief that comprehension has been attained when, in fact, comprehension has failed. In the present experiment, the illusion was defined operationally as having occurred when readers who failed to find a contradiction in a text rated their comprehension of the text as high. Texts containing contradictions between adjacent sentences were presented, and readers were explicitly asked to search for contradictions. The frequency of illusions was greater when the contradictory sentences came at the end of three-paragraph texts rather than at the end of one-paragraph texts and when the contradictory information was syntactically marked as new. These results are interpreted within a framework that emphasizes that the goal of reading expository text is to establish coherence within and among sentences. In addition, the results are apparently incompatible with the notion that readers engage in active and accurate on-line monitoring of the degree to which this goal is met.
\end{abstract}

This article examines an illusion that occurs during attempted comprehension of a text. Like illusions in perception, the discrepancy involves a mismatch between a subjective assessment and an objective fact. In the present case, the discrepancy is between self-assessment of understanding and objective accuracy of understanding. In particular, we will be concerned with self-assessments that demonstrably overestimate understanding. We call this discrepancy the illusion of knowing. As in perceptual illusions, a victim of the illusion of knowing is unlikely to be spontaneously aware that the selfassessment is illusory.

Discrepancies between self-assessment and objective understanding are commonly reported by students, particularly when they discover they have performed poorly on an exam for which they felt well prepared. Of course, students' professions of puzzlement concerning poor performance cannot be fully credited as reflecting the illusion of knowing. Face-saving, delusion, and dissembling may account for a considerable number of these expressions of surprise. However, our experience as teachers suggests that even when these cases are set aside, many genuine illusions remain. If our impressions

This research was funded in part by a grant from the Spencer Foundation to the School of Education of the University of Wisconsin, Madison, and by the National Institute of Education Grant NIE-G-81-0009 to the Wisconsin Center for Education Research. Any opinions, findings, and conclusions or recommendations expressed in this publication are those of the authors and do not necessarily reflect the views of the National Institute of Education or the Department of Education. We thank Deborah Franks, Joel Huffman, Tim Neuman, and Patricia Register for helping to write the texts and for collecting the data. Requests for reprints may be sent to the authors at the Psychology Department, University of Wisconsin, 1202 West Johnson Street, Madison, Wisconsin 53706. as teachers are correct, the illusion may be a major obstacle to effective learning and instruction.

Our research on the illusion of knowing was an effort to subject these impressions to controlled study. The efforts were successful in three respects. First, preliminary results demonstrated that the illusion does in fact occur, that it can be induced in the laboratory, and that it is remarkably common. Second, we found that the illusion can be induced more frequently in a late paragraph than in an initial one. Third, the experiment also showed that the frequency of the illusion is modulated by syntactic variables.

We used a contradiction paradigm. Subjects read an expository text and, while reading, used a rating scale to assess the degree to which they understood the text. Sometimes a sentence in the text contradicted information previously presented in the text. An illusion of knowing was operationally defined as a rating that the material with the contradictory sentence was comprehended. This procedure is appealing because failure to recognize a contradiction is a simple but plausible indicator of comprehension failure. At the same time, a rating of high comprehension indicates that subjects believe they have comprehended the text. Furthermore, the method rules out certain uninteresting explanations of the illusion. In particular, it rules out some explanations based on memory failure, because the whole text is accessible to the subject continuously. Related paradigms have been used by Williams, Taylor, and Granger (1981) and by Markman (1979); however, see Stein and Trabasso (in press) and Winograd and Johnson (Note 1) for evaluations of Markman's work.

In unpublished experiments, we used a 1,600-word text judged to be an appropriate introduction for freshmen to the perceptual problem of size constancy. At 
each of three junctures-early, intermediate, and late in the exposition-a key passage was highlighted and set off from the text by a row of asterisks. A sentence in the last key passage contradicted an inference derivable from the previous text. Immediately following each key passage, there was a 4-point scale on which the subjects rated their comprehension. Careful reading was encouraged and rereading was freely allowed.

Analysis of the comprehension ratings showed that subjects $(\mathrm{N}=24)$ were very confident in their comprehension of the key passages. For the early, intermediate, and late passages, respectively, $100 \%, 67 \%$, and $92 \%$ of the readers claimed to be "fairly certain" or "very certain" that they understood the passage. The ratings of the third passage were surprising because nearly all the subjects believed they comprehended material that was contradictory. By our operational definition, these subjects experienced an illusion of knowing.

In a second unpublished study, the text was the same, but we corrected some possible biases in the procedure. The instructions were revised to state that several versions of the text had been written, some easy and others difficult to understand. This information should have attenuated any reticence to admit comprehension failure. In addition, the subjects were explicitly directed to rate their comprehension of the key passages in relation to the preceding text, rather than their comprehension of the isolated passage. The subjects were also interviewed after they finished reading the text in an effort to determine whether they had, in fact, detected the contradiction but had somehow ignored or rationalized it.

In the second study, $63 \%, 47 \%$, and $95 \%$ of the subjects were confident in their understanding of the early, middle, and late key passages, respectively. Again, nearly all the subjects exhibited an illusion of knowing. Moreover, none indicated in the interview that the contradiction was detected but unreported.

Before describing the experiment, one observation should be made about these preliminary findings. The contradiction in the late passage was only a contradiction in relation to the information presented earlier. Since $63 \%$ and $47 \%$ of the subjects indicated that they understood the early and middle passages, respectively, perhaps only a few of the subjects could be expected to detect the contradiction. Our conclusion that $95 \%$ of the subjects exhibited the illusion of knowing might appear to be an exaggeration. One aspect of this observation is well taken: Certainly, subjects who do not understand the information presented early in the text will be unlikely to detect the contradiction. The illusion of knowing, however, is not simply a failure to detect the contradiction: It is a failure to detect a contradiction while having confidence that understanding is complete. In itself, the finding that every subject failed to detect the contradiction is not surprising; many texts are hard to understand. The interesting datum is that $95 \%$ of the subjects failed to detect the contradiction and at the same time believed they understood the text.
In the experiment, we modified our procedures for inducing the illusion, and we manipulated variables that affected its frequency. We used three expository texts titled "The Black Death in Medieval England," "Devaluation Inflation," and "Media Politics." An example appears in Table 1. Also, we used a different sort of contradiction. In the previous work, the contradiction was at the discourse level. It was a contradiction between an accurate but never stated inference and an inaccurate explicit statement. There were no sentences that directly contradicted one another. In the experiment described in this paper, the contradiction was at the sentential level. The contradictory material was explicitly presented in two sentences. These two sentences were adjacent and were the last sentences of the text. The instructions were also changed. Subjects were explicitly told that contradictions occurred in the texts. In fact, one of the subject's tasks was to search for these contradictions. Finally, we manipulated two variables that seemed likely to modulate the frequency of illusions. We contrasted (1) contradictions appearing in the first and only paragraph with those appearing in the last of three paragraphs, and (2) contradictions marked syntactically as expressing previously given information

Table 1

Example Passage

\section{Title: Media Politics}

\section{Optional Paragraphs:}

Political candidates rely heavily on media exposure to first attract and then sustain the favor of their constituents. Media channels transmit more political news per unit of time in the present age than ever before. The resulting blitz of political information appears to have repercussions for the political lives of America's public.

Different media organizations retain a vested interest in promoting one candidate at the expense of the others. As a result, the public is bombarded with a variety of political messages daily, all purporting to be impartial synopses of political actions, but which distort, in subtle, or sometimes blatant, ways the true nature of events. Additionally, this information onslaught makes simple discriminations between candidates difficult, and obfuscates basic issues. Achieving a clear perception of the political situation may become impossible, especially when ambiguity is heightened with the arrival of new political reports.

Critical Paragraph With Continuation Sentence:

The political behavior of the American public is becoming increasingly unpredictable due to widespread uncertainty regarding salient issues, major national concerns, and candidate discriminability. Public opinion polls report unexplained vacillations in party affiliation, as well as frequent fluctuations in candidate preference. Feelings of ambiguity and confusion that discourage the American citizen from partaking in the political process may explain the erratic changes in the opinions of the electorate.

\section{Final Sentence, Given Version:}

Feelings of ambiguity and confusion that discourage the American citizen from partaking in the political process may explain this stability in the opinions of the electorate.

Final Sentence, New Version:

An explanation of these findings is that feelings of ambiguity and confusion may discourage the American citizen from partaking in the political process, thus stabilizing political opinions. 
with those marked as presenting new information.

Our interest in these variables originated in the assumption that under the motivational conditions that prevailed in the experiment, the goal of reading is to establish coherence among the ideas presented in a text (Lockman \& Klappholtz, 1980). Coherence is established by determining possible structures, propositions, or concepts to which the current information should be attached.

One expectation based on this formulation of the goal of reading is that adding introductory paragraphs will decrease the frequency of detections of contradictions. Without introductory paragraphs, there are few options regarding the concepts to which the second of the two contradictory sentences in our texts should be attached. Attempting to develop coherence with the most likely option, the cognitive structure representing information from the first of the contradictory sentences, will make the contradiction manifest. Thus, the subject will detect the contradiction, and no illusion will occur. When introductory paragraphs are read, there is probably an increase in the number of structures to which the information in the second of the conflicting sentences can be attached. Not all of these options will result in the contradiction's becoming apparent. Hence, on this account, when subjects read more paragraphs, they are less likely to detect the contradiction and more likely to exhibit an illusion of knowing.

Concerning our second manipulation, the distinction between given and new information, our reasoning followed Haviland and Clark (1974). We assumed that readers attempt to establish coherence among sentences by using syntactic markers to divide a sentence into given information, which reaffirms something previously established, and new information. For example, definite articles (e.g., the) typically mark given information, whereas indefinite articles (e.g., a) typically mark new information. The reader is assumed to match the given information to a concept in the cognitive structure representing the text, and then the new information is appended to that concept.

According to this formulation, a contradiction is detected when the reader attempts to find a match between incoming information and previous information. If the attempted match fails because the incoming information is found to be logically at odds with previous information, the reader will realize that there is a contradiction. The critical point for our purposes is that an attempt to match is likely to be initiated when the incoming information is syntactically marked as given. When it is marked as new, however, no matching is likely to be attempted, for the simple reason that truly new information has no previous information it could match. Instead of being matched, incoming new information is simply appended to the cognitive structure representing the text, perhaps at the location of the information most recently marked as given. Lacking an attempted match, new information that contradicts the previous text will be accepted without scrutiny. The contradiction will go undetected, and an illusion of knowing will occur.

This line of reasoning is concerned with whether readers detect a contradiction. The argument does not explain how readers judge whether their understanding is good or poor. We shall defer discussion of this important limitation until the conclusion of the article.

To summarize, some preliminary studies suggested that the illusion of knowing does indeed occur and that it is amenable to experimental study. We therefore conducted an experiment investigating possible causes of the illusion. Using a contradiction paradigm, we asked subjects to find inconsistencies in short texts and to rate their comprehension of the texts. Some texts had a single paragraph containing contradictory sentences; others had the same paragraph preceded by two introductory paragraphs. Half the contradictions were syntactically marked as new; half, as given. The illusion of knowing was defined operationally as failure to detect a contradiction, coupled with a rating of high comprehension. We expected that the frequency of illusions would increase when introductory paragraphs were present and would decrease when the contradicting information was marked as given.

\section{METHOD}

\section{Materials}

For each of three topics, we constructed a three-paragraph text. The last paragraph was the critical paragraph. Its first sentence reiterated part of the passage title and was written so that it could serve to introduce the topic in the absence of the two preceding paragraphs.

There were three versions of the last sentence of the critical paragraph. In the control version, the last sentence was simply a continuation of, or conclusion drawn from, the previous sentence. The given version of the last sentence contained information syntactically marked as given that contradicted information in the preceding sentence. The given and control versions differed by only one or two content words. (Compare "the erratic changes" to "this stability" in Table 1.) The new version of the last sentence presented information that was marked as new and that contradicted information in the preceding sentence. The new versions of the last sentences consisted of two parts: premise-like information (e.g., "feelings of ambiguity and confusion may discourage the American citizen from partaking in the political process" in Table 1) and conclusions (e.g., "thus stabilizing political opinions," in Table 1). The arguments presented within the last sentences were internally consistent, but the conclusions (new information) contradicted information in the preceding sentences. The contradictory information occurred in the last phrase of the last sentence in both the given and new versions. Also, in both versions, the contradictory information contradicted the same portion of the preceding sentence.

When presented to the subjects, each sentence in the text was preceded by a number indicating the sentence's position within the text. In addition, two true-false questions were constructed for each topic.

\section{Procedure}

The subjects were tested in groups of six to nine individuals. The same two experimenters were present at each session. 
The subjects were instructed to read the texts carefully, "as if you were reading for a test. In fact, after each passage you will be tested on the content of each passage." Subjects were also told that a text could contain one or more contradictions and that they were to search for the contradictions. Upon finding a contradiction, the subjects recorded the sentence numbers of the contradictory sentences and wrote a brief explication of the contradiction. This explication was used to determine if the contradictions detected by the subjects were indeed the ones intended by the experimenters.

After reading a text, the subjects were asked to judge how well they understood the text. The judgments were made on a 4-point scale $(1=$ very little understood, $4=$ understood very well). Next, subjects were asked to indicate in writing the features that made the text easy or hard to understand. Finally, they answered the two true-false questions. These questions were included to encourage careful reading of the texts. All portions of the procedure were self-paced.

Before beginning the three experimental texts, subjects were given a copy of a practice text. This text contained two pairs of contradictory sentences, one with given information contradictory and the other with new information contradictory. The given-new distinction was not mentioned to the subjects, however. The practice text was read aloud by one experimenter, who pointed out the contradictions and explained why the sentences were contradictory.

Each subject read three texts, all one paragraph long or all three paragraphs long. One of the texts was on each of the three topics, and there was one control version, one given version, and one new version. The three versions of each text were read approximately equally often as the first, second, and third texts.

\section{Subjects}

The 94 subjects were students enrolled in introductory psychology courses who received course credit for participating in the experiment.

\section{RESULTS}

Detection of a contradiction was scored if a subject wrote the numbers of the two contradictory sentences and an appropriate explanation of the contradiction. In all but one case, the detections were appropriately justified. The joint criterion for an illusion of knowing was that a subject (1) failed to detect the contradiction but (2) indicated confidence in comprehension with a rating of 3 or 4 .

The major findings are summarized in Table 2 . The data in the table are, for each combination of one or three paragraphs and given or new versions, the number

Table 2

Detection (D) and Nondetection (N) of Contradictions by Condition and Comprehension Rating (CR), With Associated Probabilities of Illusions of Knowing (P)

One-Paragraph Condition Three-Paragraph Condition

\begin{tabular}{|c|c|c|c|}
\hline $\begin{array}{c}\text { Given } \\
(\mathrm{P}=.064)\end{array}$ & $\begin{array}{c}\text { New } \\
(\mathrm{P}=.149)\end{array}$ & $\begin{array}{c}\text { Given } \\
(\mathrm{P}=.234)\end{array}$ & $\begin{array}{c}\text { New } \\
(P=.511)\end{array}$ \\
\hline
\end{tabular}

\begin{tabular}{|c|c|c|c|c|c|c|c|c|}
\hline \multirow[b]{2}{*}{ CR } & & & & \\
\hline & D & $\mathrm{N}$ & D & $\mathrm{N}$ & D & $\mathbf{N}$ & D & $\mathbf{N}$ \\
\hline 1 & 0 & 0 & 1 & 0 & 0 & 1 & 0 & 0 \\
\hline 2 & 9 & 2 & 7 & 8 & 6 & 5 & 2 & 5 \\
\hline 3 & 22 & 3 & 17 & 7 & 13 & 6 & 11 & 14 \\
\hline 4 & 11 & 0 & 7 & 0 & 11 & 5 & 5 & 10 \\
\hline
\end{tabular}

Note $-P=$ proportion of all cases that are high confidence $(3$ and 4) and also nondetections.
Table 3

Frequency of Response According to Number of Paragraphs Read

\begin{tabular}{|c|c|c|c|c|c|c|}
\hline \multirow{3}{*}{$\begin{array}{l}\text { Number of } \\
\text { Paragraphs }\end{array}$} & \multicolumn{6}{|c|}{ Response } \\
\hline & \multicolumn{3}{|c|}{ Differential } & \multicolumn{3}{|c|}{ Similar } \\
\hline & $\mathrm{G}-\mathrm{N}+$ & $\mathrm{G}+\mathrm{N}-$ & Total & $\mathrm{G}-\mathrm{N}-$ & $\mathrm{G}+\mathrm{N}+$ & Total \\
\hline 1 & 6 & 2 & 8 & 38 & 1 & 39 \\
\hline 3 & 18 & 5 & 23 & 18 & 6 & 24 \\
\hline Total & 24 & 7 & 31 & 56 & 7 & 63 \\
\hline
\end{tabular}

Note-Each subject is classified in one and only one cell. $G=$ given; $N=$ new. $A$ " + " designates an illusion of knowing by the joint criterion; a "- "designates no illusion.

of cases in which the contradiction was discovered for passages given each of the comprehension ratings. The proportions of cases in which an illusion of knowing was scored by the joint criterion are given at the top of the table. An analysis of variance showed significantly more illusions for new than for given versions $[F(1,92)=$ $4.42, \mathrm{p}<.05]$, more for three paragraphs than for one $[F(1,92)=19.86, p<.001]$, and no interaction $[F(1,92)=2.92]$. This analysis might be considered suspect because the binary response clearly violated assumptions of normality and equal variance. We therefore report another analysis that may be less familiar but that has its assumptions satisfied.

In the second analysis, we began by classifying responses on the repeated measure in the experimental design, which was the given-new factor. Responses classified as differential came from subjects who showed the illusion on one version, given or new, but not the other; responses classified as similar had the illusion either on both versions or on neither. The differential responses showed whether the given-new manipulation affected the illusion, but data from the similar responses were neutral and, hence, uninformative regarding this manipulation. Next we tabulated the frequency of illusions $(+)$ and of nonillusions $(-)$ according to the joint criterion, in two separate 2 by 2 tables.

Regarding the given-new manipulation, for the 31 differential responses showing an effect of the manipulation (at the left of Table 3), 24 had an illusion for the new version but not for the given version $(p<.01)$.

The 2 by 2 layout for similar responses, shown at the right in Table 3, reveals that among subjects who either always or never exhibited the illusion, there were more illusions for three paragraphs $(6 / 24=25 \%)$ than for one paragraph $(1 / 39=3 \%)\left[\chi^{2}(1)=7.57, \mathrm{p}<.01\right]$. Confirming this result, the 2 by 2 table for differential responses revealed that among the 31 subjects who exhibited a single illusion, 23 were in the three-paragraph condition, which is significantly more than half $(p<.05)$.

Finally, regarding the interaction between the givennew effect and number of paragraphs effect, the 2 by 2 table for differential responses shows that the two effects were independent $\left[\chi^{2}(1)=.03\right]$.

Before presenting an interpretation of the effects of varying the number of paragraphs and the given-new 
variation, we will consider a number of potentially troublesome concerns.

The first concern is that the greater frequency of illusions for three paragraphs may result from a criterion change. Subjects who read three paragraphs may be forced to make many more decisions regarding possible contradictory sentences. To avoid false positives, these subjects may have raised their criteria for deciding to call a perceived inconsistency a contradiction. These high criteria could produce misses of some of the experimenterdefined contradictions. This concern was tested by examining the subjects' responses to control texts. If subjects who read three paragraphs have high criteria, their false positive rates on control texts should be lower than the false positive rates of subjects who read oneparagraph texts. The relative frequencies of false positives were .17 and .30 in the one- and three-paragraph conditions, respectively. These data are contrary to the criterion change concern, but they are confounded with the number of sentences that could produce false positives.

A critical location false positive was defined as an indication of a contradiction in the last or second-tolast sentences of control texts. Thus, the number of opportunities for making critical location false positives was the same in all conditions. The proportions of critical location false positives were .15 and .19 in the one- and three-paragraph conditions, respectively. Thus, there is no evidence that subjects set higher criteria and therefore had fewer false positives in the three-paragraph condition.

A second concern involves generalizability over texts. Of the six texts (three topics by given or new versions), five showed an increase (.11 to .63) in the proportion of illusions with more paragraphs. The one decrease was small (-.06). Similarly, the proportion of illusions was greater for the new than for the given version of five texts, with differences ranging from .12 to .38 . The single reversal was small $(-.01)$. Thus, the effects were consistent over texts.

A third concern is how the number of paragraphs might have affected the reading strategies of individual subjects. Some subjects may have been conscientious readers and detected all contradictions, whereas others may have read too casually to detect any contradictions. Thus, the number of paragraphs may have changed the number of careful readers, with fewer subjects in the three-paragraph condition reading conscientiously. In this case, we would expect most subjects to fall into one of two categories, detecting both contradictions (reading conscientiously) or detecting no contradictions (reading casually). This possibility can be tested by comparing observed frequencies of zero, one, and two detections to frequencies expected by a binomial process. Using the data reported earlier in Table 3 , we found that in the one-paragraph condition the observed (and expected, given that the probability of an illusion was .106) numbers of subjects having zero, one, and two illusions were
$38(37.53), 8(8.94)$, and $1(.53)$, respectively $\left[\chi^{2}(1)=\right.$ $.52]$. In the three-paragraph condition, the corresponding frequencies (using a probability of .372 to compute the expected frequencies) were 18 (18.52), 23 (21.97), and $6(6.52)\left[\chi^{2}(1)=.10\right]$. Since the data conformed to the binomial expectations, there was no support for the argument that the subjects were either conscientious or not and thus detected either all the contradictions or none.

In summary, marking the contradicting information as given increased the frequency of illusions, as did increasing the number of paragraphs. The increase in illusions was consistent across texts, and it reflected a decrease in the individual subjects' detection rates, rather than a change in criterion or a change in the proportion of conscientious readers.

\section{DISCUSSION}

This experiment demonstrates the high incidence of the illusion of knowing and suggests its generality across expository texts. Under the conditions instantiated here, much of what is judged to be comprehended is not.

Note that the illusions occurred under conditions that might have been expected to inhibit the occurrence of illusions. The subjects were explicitly instructed to search for textual errors and they still failed to detect up to $51 \%$ of them (see Table 2). Additionally, the contradiction was not concealed behind a veil of inferential reasoning. Contradictions between adjacent sentences were missed.

The increase in illusions when the introductory paragraphs are included in the texts and when the contradictory information is conveyed by information syntactically marked as new is compatible with our postulation that the goal of reading expository text is to establish coherence among the ideas presented in the text. Contradictions go undetected when the contradictory information is appended to a segment of the cognitive representation of the text that does not directly conflict with the contradictory information. The matching operation described in the introduction provides an explicit account of a way in which this occurs when contradictory information is marked as new. In these cases, the new information is appended to a segment of the cognitive structure that represents not contradicting information, but given information presented along with the new information.

The paragraph effect conformed to the expectations we developed in the introduction. Nevertheless, the effect of number of paragraphs could well be different under different circumstances. In particular, the paragraph effect may be conditional on the level of coherence adopted by the reader as the goal.

The instructions administered in the present experiment regarding the true-false test may have encouraged the reader to settle on a low level of coherence (e.g., coherence among the sentences). If the readers had been led to anticipate an essay examination, they might have 
adopted a higher level of coherence as a goal (e.g., developing an organizational framework). Under these circumstances, introductory paragraphs preceding the paragraph with contradictory sentences might help to activate appropriate schemata (cf. Bransford \& Johnson, 1972 ) or to introduce an organizational framework (Ausubel, 1963) to aid in detecting the contradiction. ${ }^{1}$ For these readers, contrary to the observed effects, the frequency of illusions could be lower when introductory paragraphs are read than when they are not.

Our formulation can account for one facet of the illusion of knowing, the failure to detect contradictions. It fails, however, to explain why readers mistakenly claim confidence in their understanding of the text. The frequent occurrence of the illusion of knowing is inconsistent with an assumption of active, accurate, on-line comprehension monitoring as the source of this confidence. Instead, we propose that confidence arises from a default assumption made by readers. That is, in dealing with expository text, a reader assumes by default that comprehension is progressing smoothly, and this assumption is maintained until the reader is alerted otherwise by an error signal arising from a failure in some comprehension process. For example, encountering an unfamiliar technical term in a science text will generate an error signal. Our interpretation of the effect of the givennew manipulation suggests that a mismatch between given information and the cognitive representation of the text also will generate an error signal.

Readers may favor the comprehension-monitoring procedure afforded by the default assumption on grounds of economy of processing. No special processes need be developed or activated to assess comprehension. Importantly, the process of waiting for an error signal can operate in parallel with comprehension processes, while requiring little, if any, capacity. Readers need not interrupt comprehension processes to assess their efficacy. Also, except for texts in unfamiliar domains, the assumption of comprehension is often reasonable. Unfortunately, for students and educators, it may be that when reading in new domains, our ability to detect ignorance is most impoverished. On such occasions, the illusion of knowing becomes an obstacle to effective learning and instruction.

\section{REFERENCE NOTE}

1. Winograd, P., \& Johnston, P. Comprehension monitoring and the error detection paradigm (Tech. Rep. 153). University of Illinois, Center for the Study of Reading, 1980.

\section{REFERENCES}

Ausubel, D. P. The psychology of meaningful verbal learning. New York: Grune \& Stratton, 1963.

Bransford, J. D., \& Johnson, M. K. Contextual prerequisites for understanding: Some investigations of comprehension and recall. Journal of Verbal Learning and Verbal Behavior, 1972, 11, 717-726.
Haviland, S. E., \& Clark, H. H. What's new: Acquiring new information as a process in comprehension. Journal of Verbal Learning and Verbal Behavior, 1974, 13, 512-521.

Lockman, A., \& KLApPholtz, A. D. Toward a procedural model of contextual reference. Discourse Processes, 1980, 3, 25-71.

Markman, E. M. Realizing that you don't understand: A preliminary investigation. Child Development, 1977, 48, 986-992.

Strein, N. L., \& Trabasso, T. What's in a story: An approach to comprehension and instruction. In R. Glaser (Ed.), Advances in the psychology of instruction (Vol. 2). Hillsdale, N.J: Erlbaum, in press.

Williams, J. P., Taylor, M. B., \& Granger, S. Text variations at the level of the individual sentence and the comprehension of simple expository paragraphs. Journal of Educational Psychology, 1981, 73, 851-865.

\section{NOTE}

1. Inasmuch as the critical paragraph in the experiment was written to be complete in freestanding form, the difference between three- and one-paragraph texts in this respect is not as marked as it might be ordinarily.

\section{APPENDIX \\ ADDITIONAL CRITICAL PARAGRAPHS}

\section{Title: Devaluation Inflation}

Critical paragraph with continuation sentence. One effect of devaluation of the dollar has been price inflation of both goods which we import and those which we export. Since devaluation has reduced the worth of the dollar compared to foreign currencies, we must pay more dollars for the goods which we import. Similarly, because of currency devaluation our exported goods are cheaper to foreigners, increasing their demand for those goods. U.S. manufacturers have been forced to increase domestic prices of exportable goods in response to this increased foreign demand.

Final sentence, given version. U.S. manufacturers have been forced to increase the domestic prices of exportable goods in response to this limited foreign demand.

Final sentence, new version. In response to changing economic conditions, U.S. manufacturers have been forced to cut back inventories, thus increasing delivery time and limiting foreign demand for exportable goods.

\section{Title: The Black Death in Medieval England}

Critical paragraph with continuation sentence. Mortality due to the Black Death was between $25 \%$ and $28 \%$ of medieval England. In some villages nearly all the inhabitants perished. Some areas were so depopulated that they reverted to wasteland. One result of the disease was that the surviving landlords found themselves with a markedly reduced labor force of serfs. A second result was that the serfs were able to win their freedom because of the increased demand for their services brought about by the decrease in their number.

Final sentence, given version. A second result was that the serfs were able to win their freedom because of the decrease in the demand for their services brought about by the increase in their number.

Final sentence, new version. Because the disease also destroyed much of the nobility, the feudal bond between serfs and lords was broken, a class of mobile workers was created, and thus there was an increase in the number of available workers.

(Received for publication April 28, 1982; revision accepted August 12, 1982.) 